\title{
COVID-19 Pandemie in Österreich - Zufriedenheit der Bürger*innen mit Maßnahmen und Kommunikation
}

\author{
Institut für Public Management \& Governance / Wirtschaftsuniversität Wien
}

April 2020

\author{
Author*innen: Jurgen Willems, Monika Knassmüller, Falk Ebinger, Katharina Dinhof, Moritz Schmid \\ Administrative Unterstützung: Lisa Sauberer \\ Institut für Public Management \& Governance (Website - Twitter - FIDES) \\ Wirtschaftsuniversität Wien \\ Welthandelsplatz 1, A-1020 Wien, Österreich \\ Email: pubmgt@wu.ac.at \\ Phone: +43/1/31336 - 4232
}




\section{Zusammenfassung}

Finanzierung: Die Studie ist Teil des Projektes Zusammenhalt von Bürger*innen in der Corona-Krise (COV20-039) des Institutes für Public Management \& Governance | WU Wien, finanziert vom Wiener Wissenschafts-, Forschungs- und Technologiefonds | WWTF.

Zielsetzung: Die repräsentative Umfrage (1798 in Österreich lebende Personen) erhebt die subjektive Zufriedenheit mit folgenden Aspekten der 2020 COVID-19 Krise: Umgang der Bundesregierung mit der Krise; Berichterstattung über die Maßnahmen der Bundesregierung in österreichischen Medien; Umsetzung der Maßnahmen zur Bewältigung der Krise durch (Mit)Bürger*innen, Nachbar*innen, Arbeitgeber*innen, Kolleg*innen sowie eigenem Verhalten. Zudem wird untersucht, ob substantielle Unterschiede zwischen einzelnen Gruppen (geschichtet nach Alter, Geschlecht, Bundesland) bestehen.

Stichprobe und Methode: Die Stichprobe umfasst 1798 in Österreich lebende Personen und ist repräsentativ im Hinblick auf Region (Bundesland), Alter und Geschlecht. Für die Teilgruppe der unselbstständig Erwerbstätigen $(\mathrm{n}=876)$ wurden zusätzlich die Variablen Bildungsabschluss, Beruf und beruflicher Sektor erhoben. Zur Erhebung der subjektiven Zufriedenheit kam ein deutschsprachiger online-Fragebogen mit 7-stufiger Likert Skala zum Einsatz ( -3 sehr unzufrieden bis +3 sehr zufrieden; 0 als mittlere Option). Die

Items/Fragen beginnen einheitlich mit „Wie zufrieden sind Sie damit, wie ... “ und finden sich vollständig im Anhang. Der Fragebogen enthält acht Items für die gesamte Stichprobe und weitere sechs Items für jene Befragten, die sich als unselbstständig berufstätig klassifizierten. Diese zusätzlichen Items erheben die Zufriedenheit der Befragten (a) mit Maßnahmen der Arbeitgeber*in zur Bewältigung der Krise und (b) mit dem Befolgen dieser Maßnahmen durch Kolleg*innen und die Befragten selbst. Den Befragten wurden die Items in zufälliger Reihenfolge präsentiert. Um ein gedankenloses Durchklicken durch den Fragebogen zu identifizieren, wurde in jedem Fragebogen eine Kontrollfrage als zusätzliches Item inkludiert. Bei falscher Beantwortung der Kontrollfrage wurde der betroffene Datensatz in der Auswertung nicht berücksichtigt.

Die Datenerhebung erfolgte mit Qualtrics Surveys von 17. bis 29. April 2020 und wurde von Qualtrics Panels, einem professionellen Panel-Provider durchgeführt. Ergebnisse der deskriptiven Statistik sind am Ende des Dokumentes dargestellt. Zu beachten: Die Stichprobe ist repräsentativ im Hinblick auf Bundesland, Alter und Geschlecht; darüber hinaus wurde keine Gewichtung der Daten vorgenommen. Rohdaten und Erhebungsinstrument sind öffentlich verfügbar: https://osf.io/g8hn9/

Darstellung der Ergebnisse: Die Items/Fragen sind in den Grafiken absteigend nach dem Stichprobenmittel gereiht (höchste Zufriedenheitswerte zu Beginn, niedrigste am Ende der jeweiligen Grafik). Die Mittelwerte sind in allen Grafiken durch einen Punkt im umgebenden 95\% Konfidenzintervall repräsentiert. Bei den Ergebnissen der gesamten Stichprobe und der Subgruppe der unselbstständig Erwerbstätigen sind direkt über den Konfidenzintervallen zusätzlich die numerischen Mittelwerte ausgewiesen. Alle anderen Grafiken stellen die Ergebnisse geschichtet nach demographischen Merkmalen dar.

Erkenntnisse und Schlussfolgerung: Insgesamt sind in Österreich lebende Menschen zufrieden mit den abgefragten Aspekten der COVID-19 Krise: Sämtliche Mittelwerte liegen im positiven Bereich der Skala (also über 0 auf der Skala von -3 bis +3 ). Die größte Zufriedenheit zeigen die Befragten mit sich selbst im Umgang mit der von Regierung und Arbeitgeber*in gesetzten Maßnahmen zur Bewältigung der Krise. Das betrifft sowohl die Umsetzung als auch die Einhaltung der Maßnahmen durch die Befragten. Im Gegensatz dazu ist die Zufriedenheit am geringsten, wenn es um die Befolgung der Maßnahmen durch Nachbarn und andere Bürger*innen sowie um die Berichterstattung über die Maßnahmen in den österreichischen Medien geht. Die Schichtung nach Geschlecht, Alter, Bundesland, Bildungsabschluss, Beruf oder beruflichem Sektor zeigt geringe, aber keine bedeutenden Unterschiede zwischen den jeweiligen Untergruppen. 


\section{Bürger*innen}

\subsection{Zufriedenheitswerte gesamt}

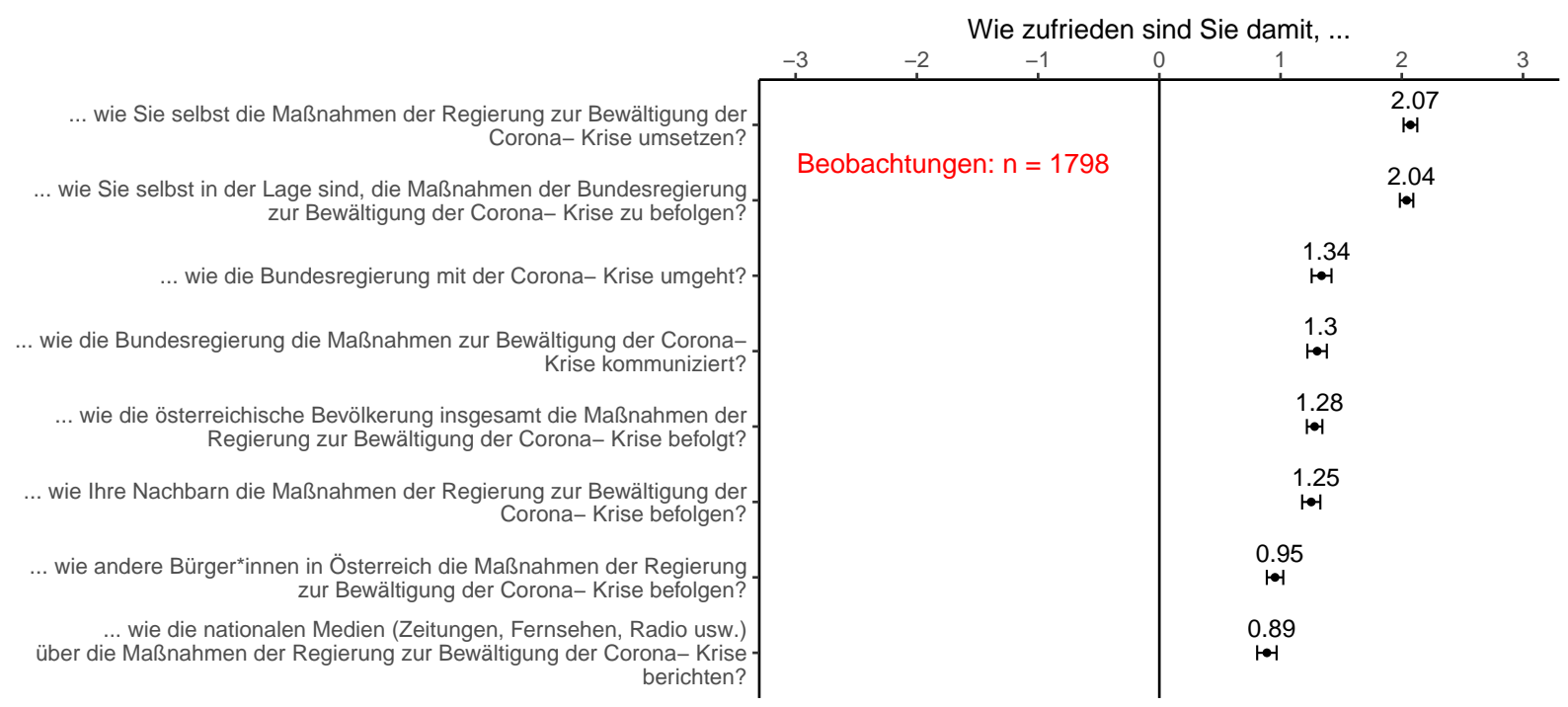

Insgesamt sind sämtliche Durchschnittswerte im positive Bereich. Die größte Zufriedenheit herrscht unter den Befragten mit der eigenen Befolgung und Umsetzung der Regierungsmaßnahmen zur Bewältigung der COVID-19 Krise. Deutlich geringer - wenn auch noch immer positiv - ist die Zufriedenheit mit der Regierung und anderen (Mit)Bürger*innen, während die Berichterstattung in nationalen Medien (Zeitungen, Fernsehen, Radio, etc) die im Vergleich geringste Zustimmung erfährt.

Download Grafik: https://doi.org/10.6084/m9.figshare.12217991 


\subsection{Zufriedenheit nach Alter}

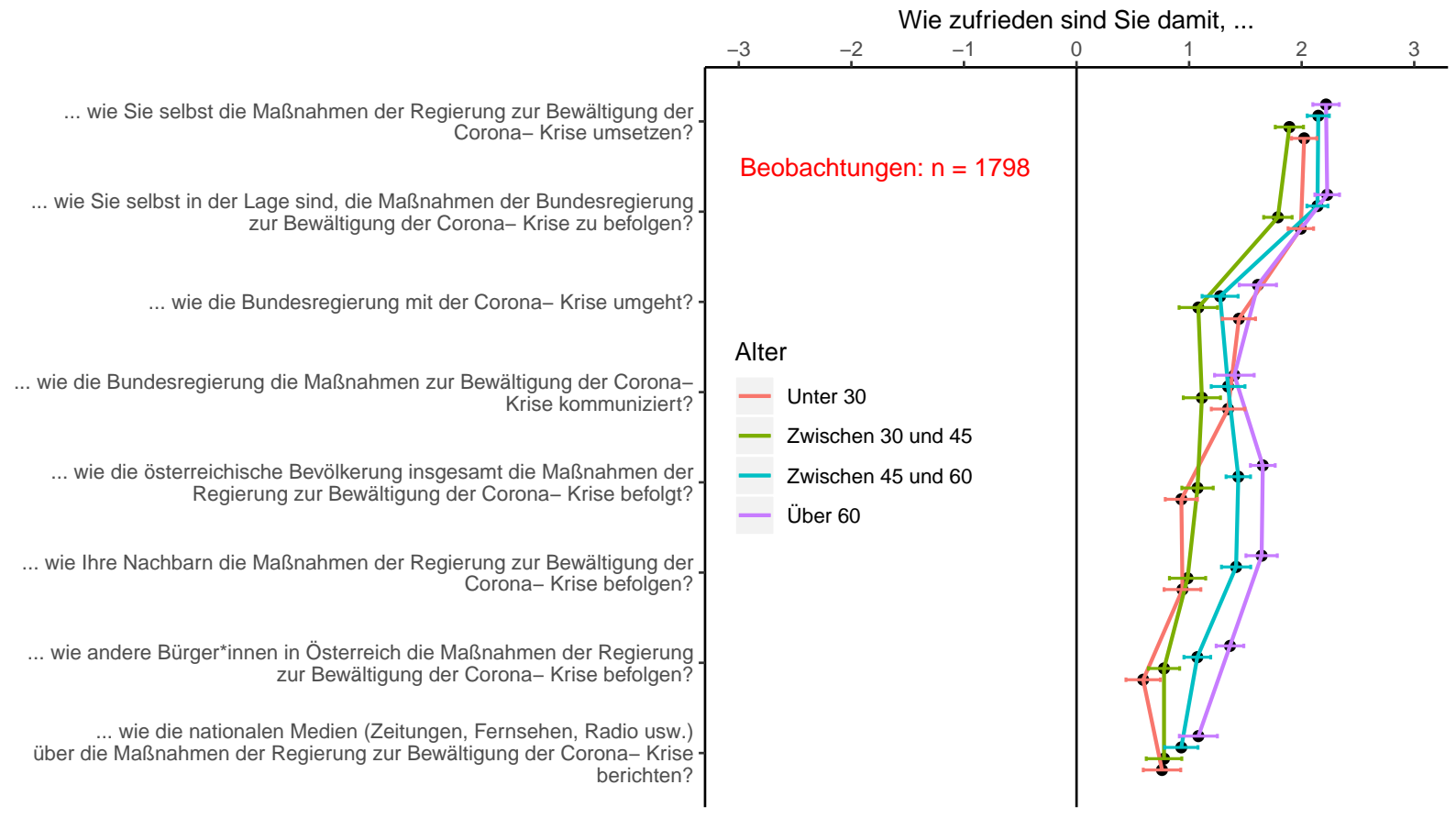

Die Splittung nach Altersgruppen zeigt, dass je älter die Befragten sind, desto zufriedener sind sie mit der Befolgung der COVIC-19 Maßnahmen durch andere (Mit)Bürger*innen / die Bevölkerung.

Download Grafik: https://doi.org/10.6084/m9.figshare.12218021 


\subsection{Zufriedenheit nach Geschlecht}

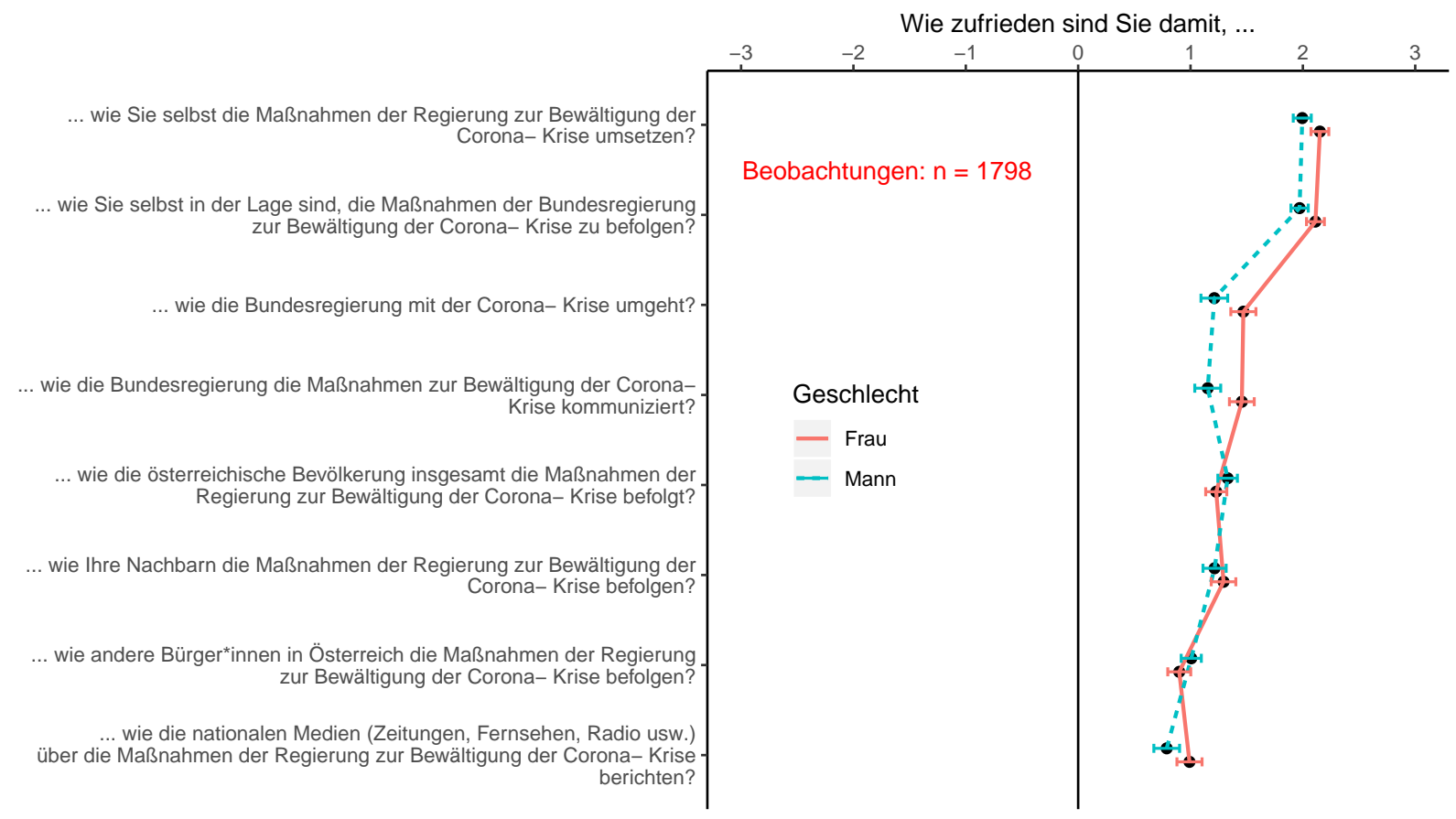

Unterschiede zwischen Männern und Frauen sind nicht oder nur in geringem Ausmaß festzustellen: So sind Frauen etwas zufriedener mit dem eigenen Verhalten sowie mit der Regierung (sowohl hinsichtlich Maßnahmen als auch deren Kommunikation).

Download Grafik: https://doi.org/10.6084/m9.figshare.12218045 


\subsection{Zufriedenheit nach Bundesland}

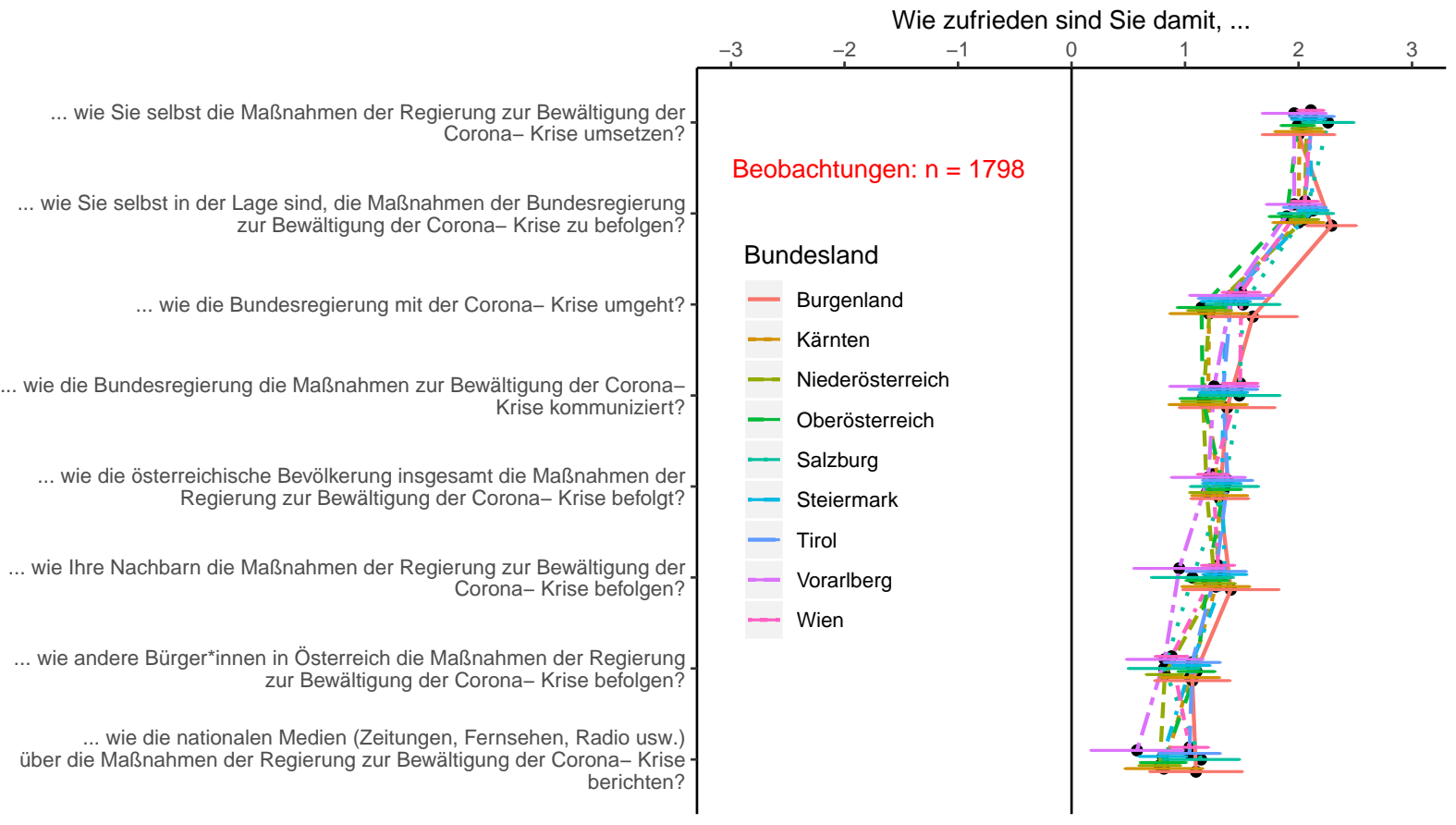

Nicht zuletzt aufgrund der relativ breiten Konfidenzintervalle sind keine nennenswerten Unterschiede feststellbar.

Download Grafik: https://doi.org/10.6084/m9.figshare.12218054 


\subsection{Zufriedenheit nach Beschäftigung}

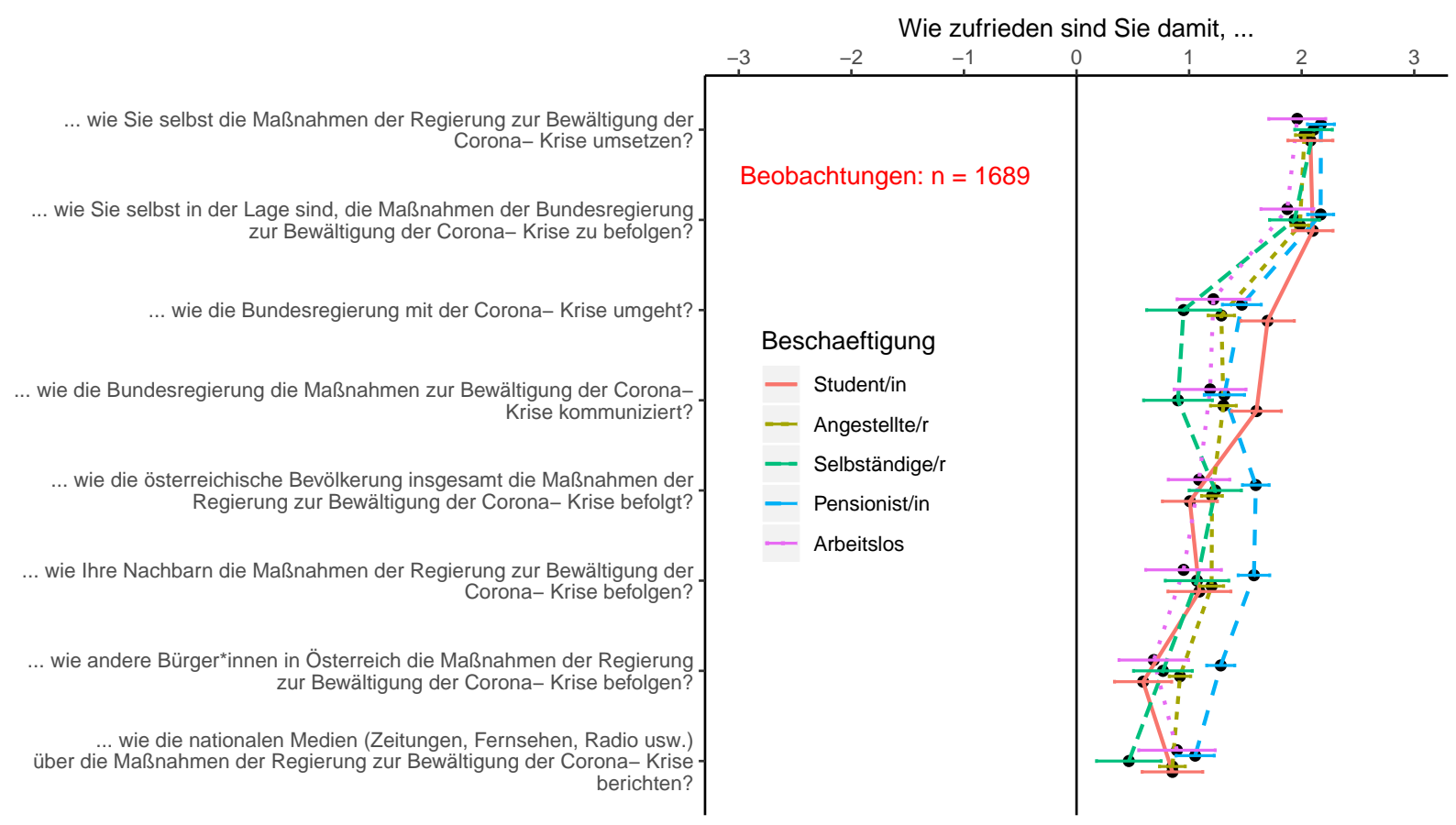

In Übereinstimmung mit der Auswertung nach Altersgruppen weisen Pensionist*innen die höchsten Zufriedenheitswerte mit der Umsetzung der COVID-19 Maßnahmen durch andere / die Bevölkerung auf. Mit der Regierung, sowohl hinsichtlich des Umgangs mit der Krise als auch die Kommunikation der Maßnahmen betreffend, sind Selbstständige am wenigsten und Studierende am meisten zufrieden. Zu beachten ist allerdings, dass die Datensammlung vor der zweiten Lockerung der Restriktionen für (kleine) Unternehmen durchgeführt wurde.

Befragte, die sich keiner Beschäftigungsgruppe zuordneten, sind in dieser Grafik nicht berücksichtig.

Download Grafik: https://doi.org/10.6084/m9.figshare.12218063 


\subsection{Zufriedenheit nach Bildungsniveau}

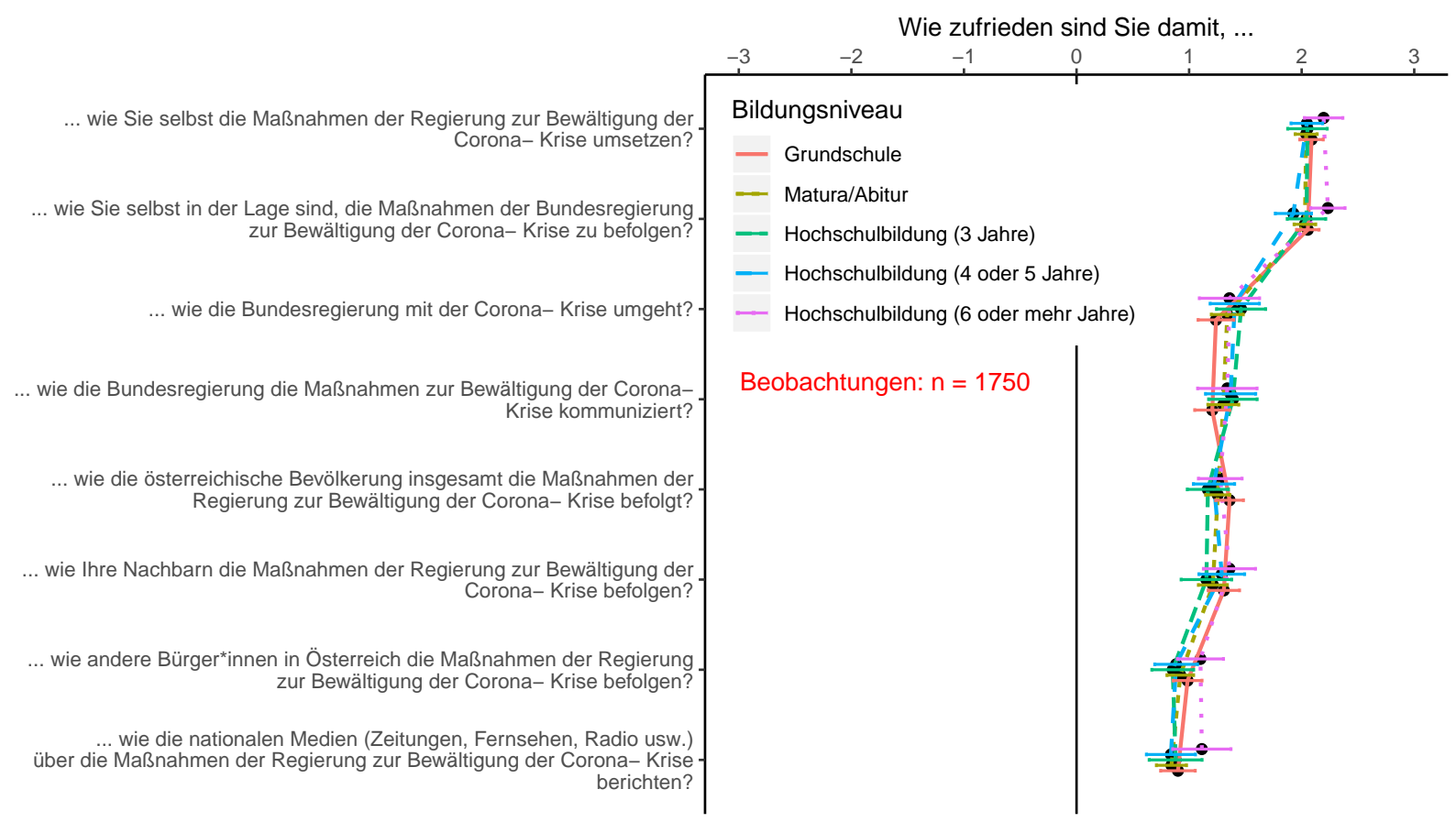

Keine nennenswerten Unterschiede nach Bildungsniveau feststellbar. Befragte, die keine Angaben zum Bildungsabschluss machten, sind in dieser Grafik nicht berücksichtig.

Download Grafik: https://doi.org/10.6084/m9.figshare.12218069 


\section{Unselbständig Erwerbstätige $(\mathrm{n}=876)$}

\subsection{Zufriedenheitswerte gesamt}

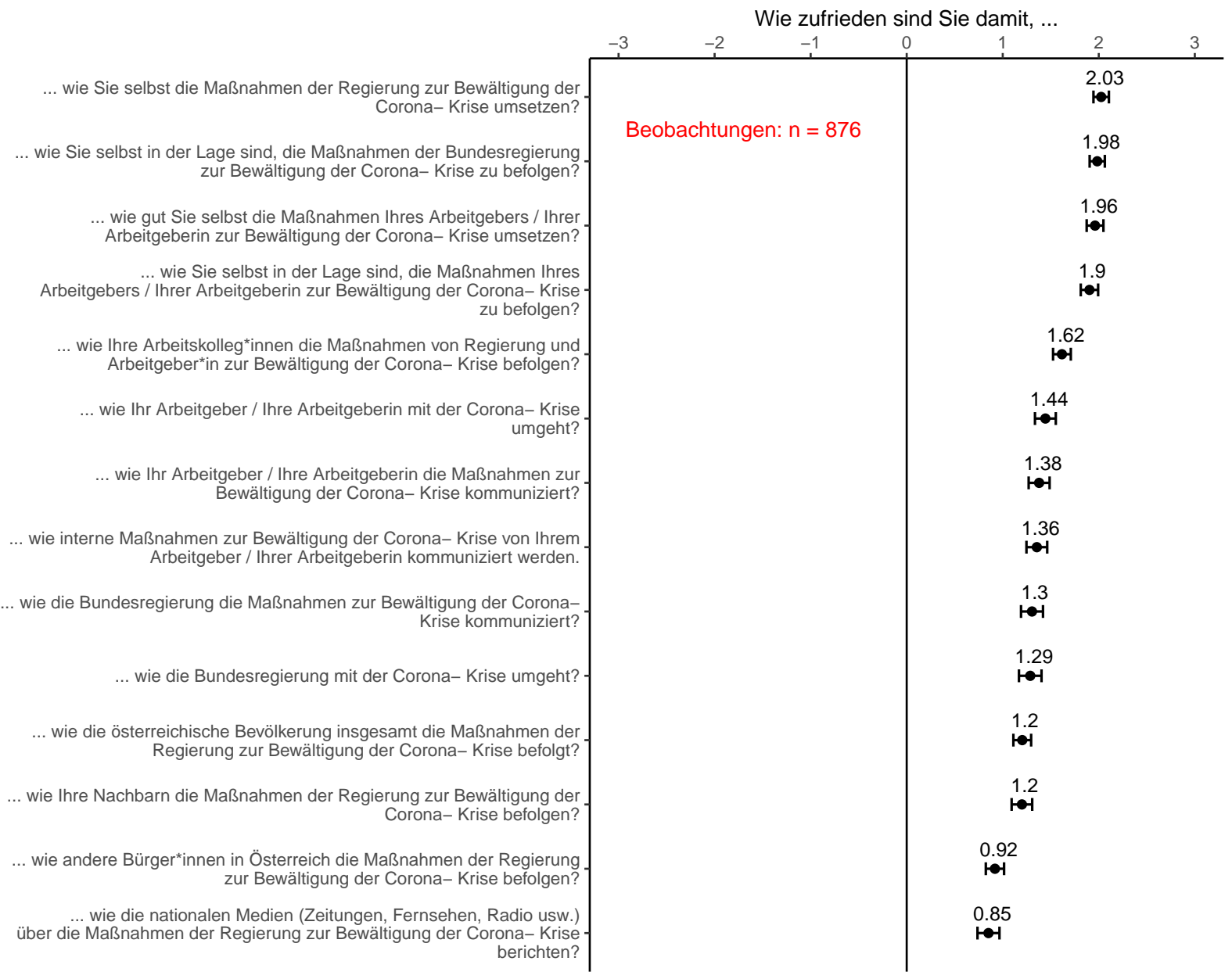

Die Darstellung der Gruppe der unselbständig Erwerbstätigen sind wie die bisherigen Grafiken absteigend nach Mittelwert gereiht, ergänzt um die Zufriedenheit mit Arbeitgeber*in und Kolleg*innen. Diese zusätzlichen Items reihen sich zwischen den bisherigen Extremen ein und bestätigen die in der gesamten Stichprobe festgestellten Unterschiede zwischen der Zufriedenheit mit eigenem Verhalten und jenem anderer Personengruppen (Nachbarn, Bürg*innen, Bevölkerung).

Download Grafik: https://doi.org/10.6084/m9.figshare.12218075 


\subsection{Zufriedenheit nach Sektor}

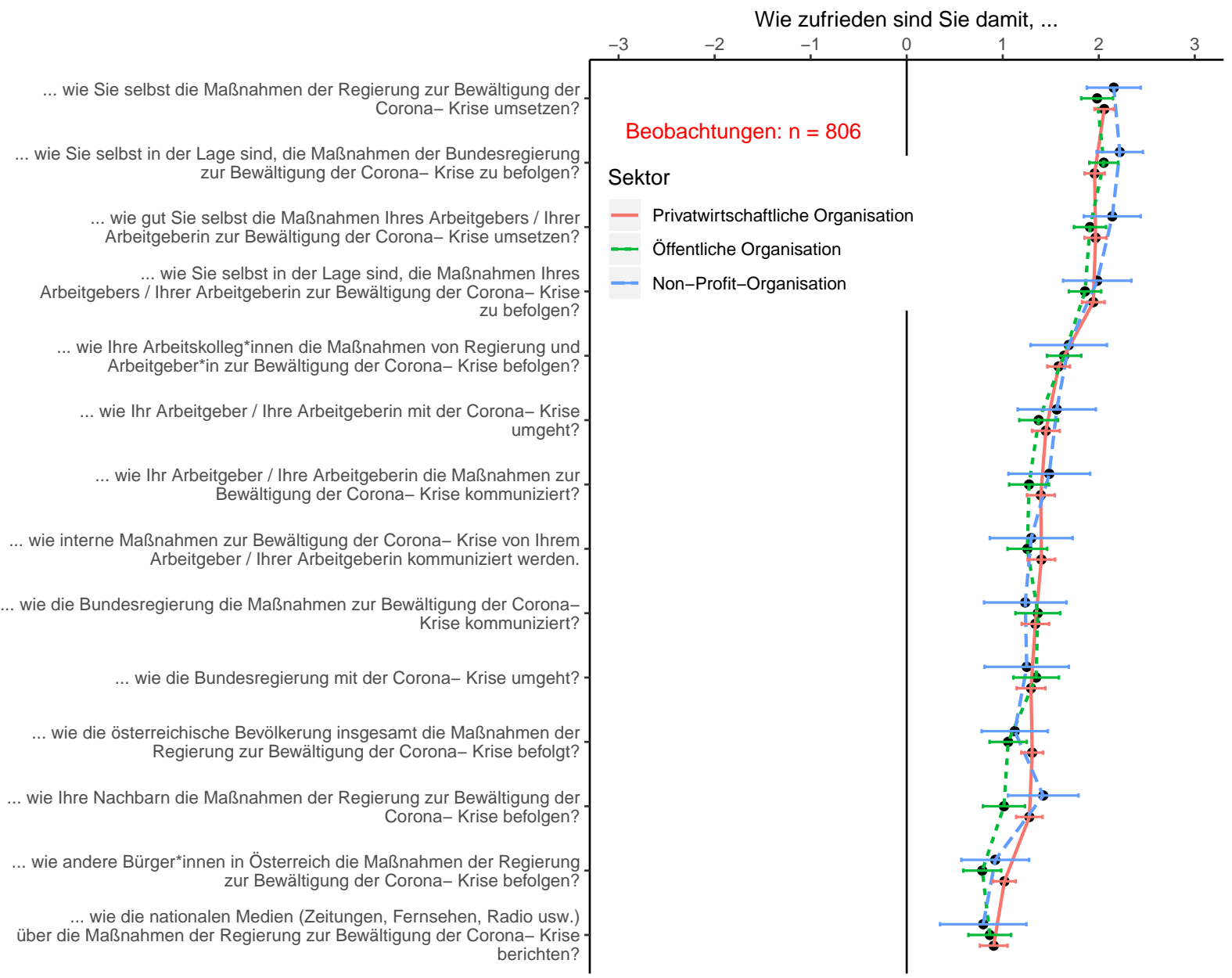

Für die Subgruppe der unselbständig Erwerbstätigen, die Angaben zum Sektor gemacht haben $(\mathrm{n}=806)$, können keine bedeutenden sektorspezifischen Unterschiede in den Zufriedenheitswerten festgestellt werden.

Download Grafik: https://doi.org/10.6084/m9.figshare.12218084 


\subsection{Zusatzitems für die Subgruppe unselbstständig Erwerbstätiger}

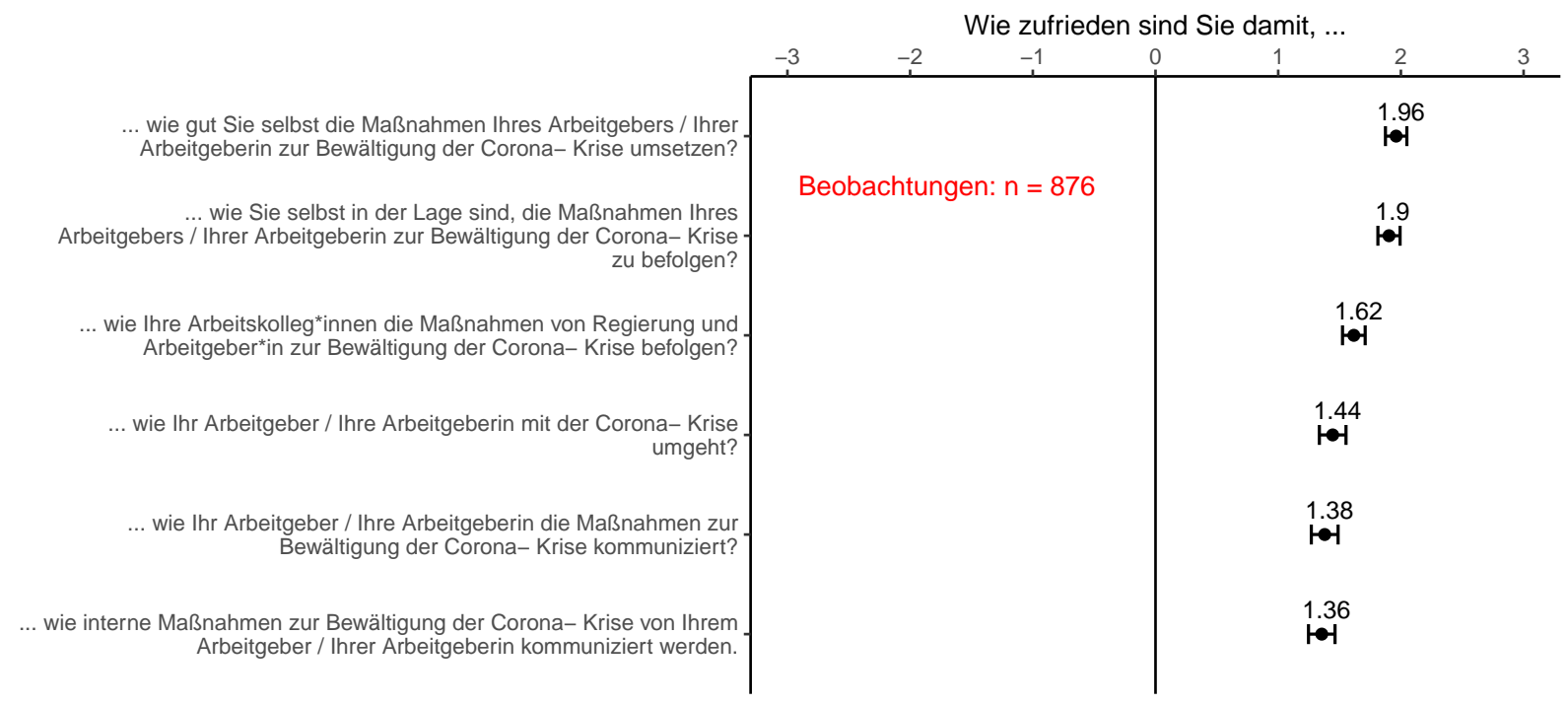

In der Grafik sind die Ergebnisse der Zusatzitems für die Gruppe der unselbstständig Erwerbstätigen, die das Arbeitsumfeld fokussieren (Selbstbeschreibung, Arbeitgeber*in, Kolleg*innen), noch einmal gesammelt dargestellt.

Download Grafik: https://doi.org/10.6084/m9.figshare.12218111 


\section{Extras}

\subsection{Items}

\begin{tabular}{|c|c|}
\hline Variables & Items \\
\hline OverallSatisfaction_01 & ... wie die Bundesregierung mit der Corona- Krise umgeht? \\
\hline OverallSatisfaction_02 & $\begin{array}{l}\text {... wie die Bundesregierung die Maßnahmen zur Bewältigung der } \\
\text { Corona- Krise kommuniziert? }\end{array}$ \\
\hline OverallSatisfaction_03 & $\begin{array}{l}\text {... wie die nationalen Medien (Zeitungen, Fernsehen, Radio usw.) } \\
\text { über die Maßnahmen der Regierung zur Bewältigung der Corona- } \\
\text { Krise berichten? }\end{array}$ \\
\hline OverallSatisfaction_04 & $\begin{array}{l}\text {.. wie Ihre Nachbarn die Maßnahmen der Regierung zur Bewältigung } \\
\text { der Corona- Krise befolgen? }\end{array}$ \\
\hline OverallSatisfaction_05 & $\begin{array}{l}\text {... wie andere Bürger*innen in Österreich die Maßnahmen der } \\
\text { Regierung zur Bewältigung der Corona- Krise befolgen? }\end{array}$ \\
\hline OverallSatisfaction_06 & $\begin{array}{l}\text {.. wie die österreichische Bevölkerung insgesamt die Maßnahmen der } \\
\text { Regierung zur Bewältigung der Corona- Krise befolgt? }\end{array}$ \\
\hline OverallSatisfaction_07 & $\begin{array}{l}\text {.. wie Sie selbst in der Lage sind, die Maßnahmen der } \\
\text { Bundesregierung zur Bewältigung der Corona- Krise zu befolgen? }\end{array}$ \\
\hline OverallSatisfaction_08 & $\begin{array}{l}\text {... wie Sie selbst die Maßnahmen der Regierung zur Bewältigung der } \\
\text { Corona- Krise umsetzen? }\end{array}$ \\
\hline OverallSatisfaction_09 & $\begin{array}{l}\text {... wie Ihr Arbeitgeber / Ihre Arbeitgeberin mit der Corona- Krise } \\
\text { umgeht? }\end{array}$ \\
\hline OverallSatisfaction_10 & $\begin{array}{l}\ldots \text { wie Ihr Arbeitgeber / Ihre Arbeitgeberin die Maßnahmen zur } \\
\text { Bewältigung der Corona- Krise kommuniziert? }\end{array}$ \\
\hline OverallSatisfaction_11 & $\begin{array}{l}\text {... wie interne Maßnahmen zur Bewältigung der Corona- Krise von } \\
\text { Ihrem Arbeitgeber / Ihrer Arbeitgeberin kommuniziert werden. }\end{array}$ \\
\hline OverallSatisfaction_12 & $\begin{array}{l}\text {... wie Ihre Arbeitskolleg*innen die Maßnahmen von Regierung und } \\
\text { Arbeitgeber*in zur Bewältigung der Corona- Krise befolgen? }\end{array}$ \\
\hline OverallSatisfaction_13 & $\begin{array}{l}\text {... wie Sie selbst in der Lage sind, die Maßnahmen Ihres Arbeitgebers } \\
\text { / Ihrer Arbeitgeberin zur Bewältigung der Corona- Krise zu befolgen? }\end{array}$ \\
\hline OverallSatisfaction_14 & $\begin{array}{l}\ldots \text { wie gut Sie selbst die Maßnahmen Ihres Arbeitgebers / Ihrer } \\
\text { Arbeitgeberin zur Bewältigung der Corona- Krise umsetzen? }\end{array}$ \\
\hline
\end{tabular}




\subsection{Korrelation (Employee subset; $\mathrm{n}=876$ )}

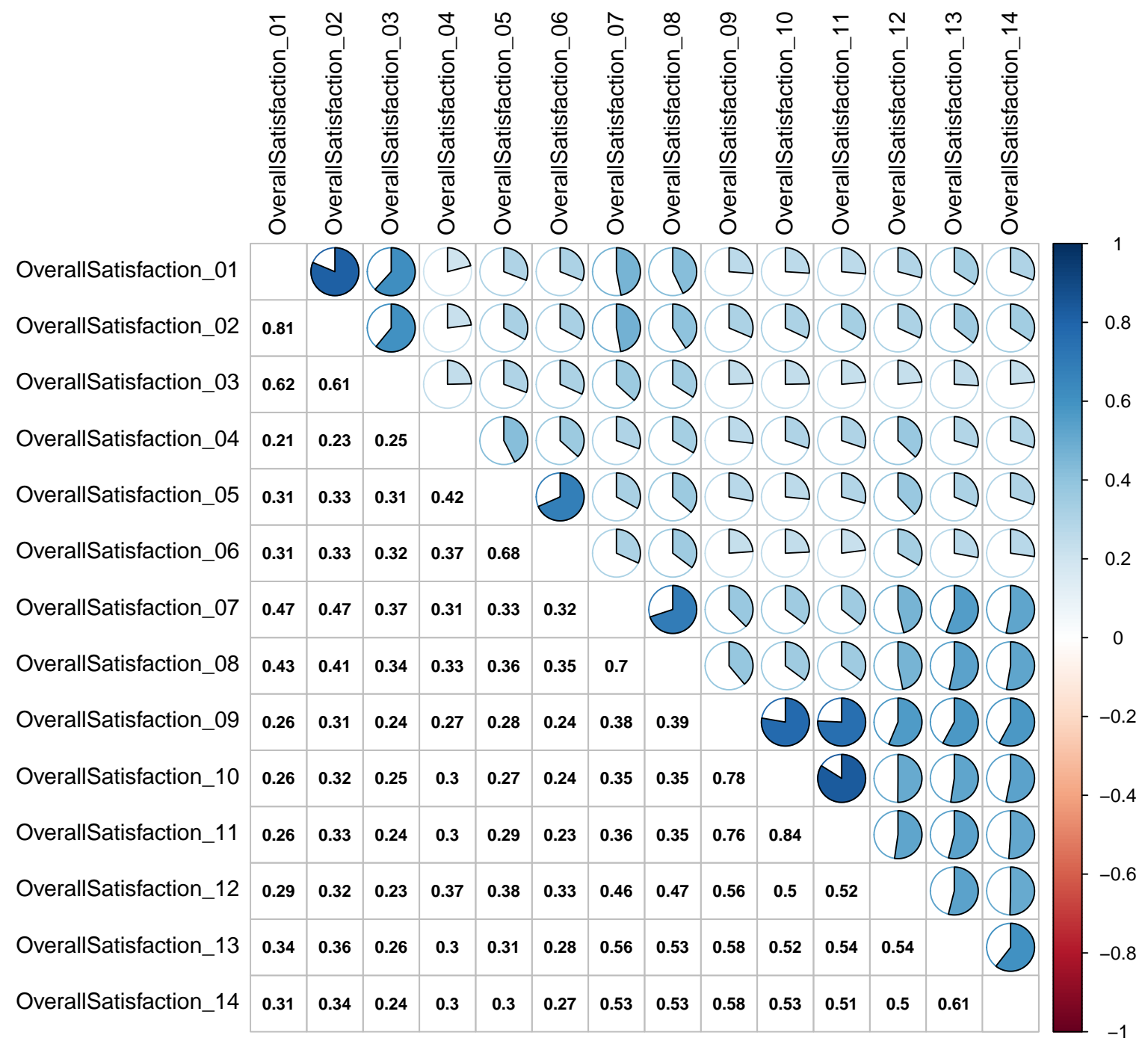




\subsection{Descriptives}

\begin{tabular}{|c|c|}
\hline & DATA $(\mathrm{N}=1,798)$ \\
\hline \multicolumn{2}{|l|}{ Alter } \\
\hline Unter 30 & $407(22.64 \%)$ \\
\hline Zwischen 30 und 45 & $440(24.47 \%)$ \\
\hline Zwischen 45 und 60 & $563(31.31 \%)$ \\
\hline Über 60 & $388(21.58 \%)$ \\
\hline \multicolumn{2}{|l|}{ Geschlecht } \\
\hline Weiblich & $878(48.83 \%)$ \\
\hline Männlich & $920(51.17 \%)$ \\
\hline \multicolumn{2}{|l|}{ Bundesland } \\
\hline Burgenland & $62(3.45 \%)$ \\
\hline Carinthia & $118(6.56 \%)$ \\
\hline Niederösterreich & $359(19.97 \%)$ \\
\hline Oberösterreich & $296(16.46 \%)$ \\
\hline Salzburg & $92(5.12 \%)$ \\
\hline Steiermark & $259(14.40 \%)$ \\
\hline Tirol & $128(7.12 \%)$ \\
\hline Vorarlberg & $78(4.34 \%)$ \\
\hline Wien & $406(22.58 \%)$ \\
\hline \multicolumn{2}{|l|}{ Education } \\
\hline Grundschule & $515(28.64 \%)$ \\
\hline Matura/Abitur & $625(34.76 \%)$ \\
\hline Hochschulbildung (3 Jahre) & $234(13.01 \%)$ \\
\hline Hochschulbildung (4 oder 5 Jahre) & $217(12.07 \%)$ \\
\hline Hochschulbildung (6 oder mehr Jahre) & $159(8.84 \%)$ \\
\hline 'Trifft nicht zu' & $48(2.67 \%)$ \\
\hline \multicolumn{2}{|l|}{ Hauptbeschäftigung } \\
\hline Student/in & $142(7.90 \%)$ \\
\hline Angestellte/r & $876(48.72 \%)$ \\
\hline Selbständige/r & $142(7.90 \%)$ \\
\hline Pensionist/in & $427(23.75 \%)$ \\
\hline Arbeitslos & $102(5.67 \%)$ \\
\hline \multicolumn{2}{|l|}{ Sektor } \\
\hline Privatwirtschaftliche Organisation (For-Profit-Organisation) & $512(58.45 \%)$ \\
\hline Öffentliche Organisation & $230(26.26 \%)$ \\
\hline Non-Profit-Organisation & $64(7.31 \%)$ \\
\hline Sonstiges & 922 \\
\hline
\end{tabular}

Other relevant socio-demographic resources on COVID-19 in Austria:

- WWTF COVID-19 Rapid Response Call

- Other projects at the WU, funded by WWTF COVID-19 Rapid Response Call

- Austrian Corona Panel Data (Universität Wien) 